\title{
HEREDITARILY PYTHAGOREAN FIELDS, INFINITE HARRISON-PRIMES AND SUMS OF $2^{n}$ th POWERS
}

\author{
BY EBERHARD BECKER \\ Communicated by Olga Taussky Todd, July 21, 1977
}

In [3] W. D. Geyer studied (infinite) algebraic number fields having an absolute Galoisgroup which is solvable as an abstract group. In particular he showed that for a real number field $K$ of this type the absolute Galoisgroup $G(\bar{K} \mid K(i))$ must be abelian (we denote the algebraic closure of a field $k$ by $\bar{k}$ ). Geyer's work may therefore be considered as a generalization of the well-known characterization of real-closed fields given by $\mathrm{E}$. Artin and $\mathrm{O}$. Schreier. This note reports on the work [1] originated in an attempt to carry over Geyer's results to arbitrary formally real fields $K$ (= real fields). We investigate those fields with abelian Galoisgroup $G(\bar{K} \mid K(i))$ which may be regarded as substitutes for real-closed fields. The orderings of real-closed fields are to be replaced by certain infinite Harrison-primes, and the study of sums of squares by orderings can be extended with help of these Harrison-primes to sums of $2^{n}$ th powers.

1. Hereditarily pythagorean fields. A real field $K$ is called pythagorean if $K^{2}+K^{2}=K^{2}$ holds, hereditarily pythagorean $(=\mathrm{h}$. p.) if any real algebraic extension is pythagorean. Let $\mathbf{Z}_{p}$ be the compact additive group of the $p$-adic integers, $\delta_{i j}$ the Kronecker-symbol and $\operatorname{Br}(K)$ the Brauergroup of the real field $K$.

THEOREM 1. $K$ is a h.p. field iff $G(\bar{K} \mid K(i))$ is abelian. If $K$ is h.p., then

(i) $G(\bar{K} \mid K)=\langle\sigma\rangle \times G(\bar{K} \mid K(i)), \sigma^{2}=1, \sigma$ operates by inversion on $G(\bar{K} \mid K(i))$,

(ii) $G(\bar{K} \mid K(i)) \cong \Pi_{p} Z_{p}^{\alpha} p$ with $\alpha_{p}=-\delta_{2 p}+\operatorname{dim}_{F_{p}} K^{\times} / K^{\times p}$,

(iii) $\operatorname{Br}(K)$ has exponent 2, $\operatorname{dim}_{\mathrm{F}_{2}} \operatorname{Br}(K)=\alpha_{2}+\left(\begin{array}{c}\alpha_{2}^{2} \\ 2\end{array}\right)+1$

H.p. fields can further be characterized by the Haar-measure of the set of involutions in $G(\bar{K} \mid K)$ [1], by the existence of a certain henselian valuation [2] (both due to $\mathbf{L}$. Bröcker), by the existence of a Kummer-theory for all algebraic extensions [1] (F. Halter-Koch) or by torsion properties of the Wittring of $K(X)[1]$.

2. Infinite Harrison-primes. An infinite Harrison-prime $P$ [4] of $K$ is called an ordering of type $n \in \mathrm{N}$ if $K^{2^{n}} \subset P$ and of exact type $n$ if $K^{2^{n}} \subset P$, $K^{2 n-1} \not \subset P$. Orderings of type 1 are the usual orderings. Let $Q_{n}$ be the subset of all sums of $2^{n}$ th powers in $K$. Then $Q_{n}=\bigcap P$ where $P$ ranges over all orderings of type $n$, the case $n=1$ is due to E. Artin.

AMS (MOS) subject classifications (1970). Primary 12D15, $12 \mathrm{E} 99$. 
Let $L \mid K$ be a field extension, $P, \widetilde{P}$ orderings of higher type of $K, L$ respectively. $(L, \widetilde{P})$ extends $(K, P)$ if $\widetilde{P} \cap K=P$ and $P$ and $\widetilde{P}$ have the same exact type. In [1] an extension theory is established, for example: (i) the number $r$ of extensions to $L$ is less than or equal to $[L: K]$, (ii) $r=0$ or $r=[L: K]$ if $L \mid K$ is a Galois-extension, in the latter case all extensions are conjugate. The extension-theory applies to the real closures $(R, \widetilde{P})(=$ the maximal algebraic extensions) of $(K, P)$. Let $P$ be of exact type $n \geqslant 2$, the case $n=1$ is due to E. Artin and O. Schreier.

THEOREM 2. (i) A real closure $(R, \widetilde{P})$ is a h.p. field with $G(\bar{R} \mid R(i)) \cong$ $\mathrm{Z}_{2}$, has a henselian valuation with real-closed residue-class-field, two orderings of type 1 and a single ordering of exact type $m$ for $m \geqslant 2$,

(ii) two real closures $\left(R_{i}, P_{i}\right), i=1,2$, of $(K, P)$ are isomorphic iff $R_{1}^{2^{m}}$ $\cap K=R_{2}^{2 m} \cap K$ for all $m \in \mathbf{N}$.

Different from the usual Artin-Schreier-Theory there are in general infinitely many nonisomorphic real closures of a given $(K, P)$. The proofs are essentially carried out by valuation theory since for an ordering $P$ of higher type the set $\mathcal{O}(P)=\{a \in K \ln \pm a \in P$ for some $n \in \mathrm{N}\}$ is a valuation-ring [5]. Furthermore $\boldsymbol{P}$ can be constructed from an archimedean ordering of type 1 of the residue-class-field by means of a certain character of the value-group of $\mathfrak{D}(P)$.

3. Sums of $2^{n}$ th powers. The starting points for the applications to sums of $2^{n}$ th powers are the result $Q_{n}=\bigcap P$ and the facts about $\mathrm{O}(P)$ just mentioned. Let $K$ be an infinite not necessarily real field, $n \in \mathbf{N}$.

Theorem 3. If $-1 \in Q_{1}$ (i.e. $K$ is not real), then $-1 \in Q_{n}$.

This was also proved by Joly [7].

THEOREM 4. The following statements are equivalent: (i) any valuationring of $K$ with a real residue-class-field has a 2-divisible value-group, (ii) $Q_{1}=$ $Q_{n}$ for some $n$, (iii) $Q_{1}=Q_{n}$ for all $n$.

Theorem 4 applies to number fields, more generally to algebraic extensions of a real field with a single ordering of type 1 .

THEOREM 5. For all $x_{1}, \ldots, x_{r} \in K, n, m \in \mathrm{N}$, there exist $y_{1}, \ldots, y_{s}$ $\in K$ such that

$$
\left(x_{1}^{2^{n}}+\cdots+x_{r}^{2^{n}}\right)^{2^{m}}=y_{1}^{2^{n+m}}+\cdots+y_{s}^{2^{n+m}} .
$$

Theorem 5 applied to $\mathrm{Q}\left(X_{1}, \ldots, X_{r}\right)$ generalizes in a certain sense an identity of Hilbert used in his solution of the Waring-problem [6]. 


\section{REFERENCES}

1. E. Becker, Hereditarily pythagorean fields and orderings of higher types, IMPALecture Notes, Rio de Janeiro (to appear).

2. L. Bröcker, Characterization of fans and hereditarily pythagorean fields, Math. $\mathrm{Z}$. 151 (1976), 149-163.

3. W. D. Geyer, Unendliche algebraische Zahlkörper, über denen jede Gleichung auflösbar von beschränkter Stufe ist, J. Number Theory 1 (1969), 346-374.

4. D. K. Harrison, Finite and infinite primes for rings and fields, Mem. Amer. Math. Soc. 68 (1966), 1-62.

5. D. K. Harrison and H. Warner, Infinite primes of fields and completions, Pacific J. Math. 45 (1973), 201-216.

6. D. Hilbert, Beweis für die Darstellbarkeit der ganzen Zahlen durch eine feste Zahl n-ter Potenz (Waringsches Problem), Math. Ann. 67 (1909), 281-300.

7. J.-R. Joly, Sommes des puissances d-ièmes dans un anneau commutatif, Acta Arith. 17 (1970), 37-114.

MATHEMATISCHES INSTITUT DER UNIVERSITÄT, WEYERTAL 86-90, 5000 KÖLN 41, FEDERAL REPUBLIC OF GERMANY 